\title{
Fuzzy Clusters with Volume Prototypes \\ in the Thematic Processing \\ of the Earth Remote Sensing Data
}

\author{
Aleksey A. Buchnev and Valeriy P. Pyatkin* \\ Institute of Computational Mathematics \\ and Mathematical Geophysics SB RAS \\ 6 Akademika Lavrentieva, Novosibirsk, 630090, Russia
}

Received 26.01.2017, received in revised form 14.03.2017, accepted 19.07.2017

The fuzzy clustering technology of the Earth remote sensing data, based on extended C-means and Gustafson-Kessel algorithms, is discussed. The algorithms extensions consist of clusters with volume prototypes construction and using of clusters similarity measure. The volume prototypes are less sensitive to a bias in the distribution of the data, and similar clusters are merged during clustering.

Keywords: fuzzy clustering, volume prototypes, clusters similarity, extended C-means algorithm, extended Gustafson-Kessel algorithm.

Citation: Buchnev A.A., Pyatkin V.P. Fuzzy clusters with volume prototypes in the thematic processing of the earth remote sensing data, J. Sib. Fed. Univ. Eng. technol., 2017, 10(6), 723-726. DOI: 10.17516/1999-494X-2017-10-6-723-726.

(C) Siberian Federal University. All rights reserved

* Corresponding author E-mail address: baa@ooi.sscc.ru,pvp@ooi.sscc.ru 


\title{
Нечеткие кластеры с объемными прототипами \\ в тематической обработке данных \\ дистанционного зондирования Земли
}

\author{
А.А. Бучнев, В.П. Пяткин \\ Институт вычислительной математики и \\ математической геофизики СО РАН
}

Россия, 630090, Новосибирск, пр. Академика Лаврентьева, 6

Рассматривается технология нечеткой кластеризации данных дистанционного зондирования Земли (ДЗ3) расширенныли алгоритмами С-средних и Густафсона-Кесселя. Расширения алгоритмов состоят в использовании объемных прототипов и меры сходства кластеров. Объемные прототипь менее чувствительны к иумовым выбросам в распределении данных. Кроме того, использование меры сходства позволяет объединять кластеры в процессе кластеризации.

Ключевые слова: нечеткая кластеризация, объемные прототипь, сходство кластеров, расширенный алгоритм С-средних, расширенный алгоритм ГустафсонаКесселя.

Одним из основных вопросов тематической обработки (интерпретации) данных ДЗЗ является вопрос повышения качества распознавания. Возникающие трудности обусловлены, в частности, «загрязнением» выборок смешанными векторами признаков, т.е. векторами, которые образуются при попадании в элемент разрешения съемочной системы нескольких природных объектов $[1,2]$. Большинство алгоритмов классификации для отнесения векторов признаков классам вычисляют для каждого вектора значения подходящей функции «правдоподобия». В случае зачисления вектора признаков в класс по максимальному значению функции правдоподобия получается так называемая жесткая кластеризация (например, так работает классический алгоритм $K$-средних).

Альтернативным подходом служит нечеткая кластеризация, которая позволяет каждому вектору признаков принадлежать одновременно всем кластерам с определенной степенью членства (принадлежности) в каждом кластере. Одним из первых алгоритмов нечеткой кластеризации является, вероятно, алгоритм $C$-средних [3]. Вопросы реализации и использования этого алгоритма в обработке данных ДЗ3 освещаются в [4].

Дальнейшим развитием системы нечеткой кластеризации данных ДЗ3 выступает реализация нечеткой кластеризации расширенными алгоритмами C-средних (Fuzzy C-means - FCM) и Густафсона-Кесселя (Gustafson-Kessel - GK) [5]. В алгоритме FCM выбранная метрика, определяющая форму получаемых кластеров, одинакова для всех кластеров и не меняется в процессе работы. Принципиальное отличие алгоритма $G K$ от алгоритма $F C M$ состоит в том, что каждый кластер имеет индивидуальную метрику, основанную на нечеткой ковариационной матрице кластера (метрика Махаланобиса). Эта метрика динамически меняется в процессе выполнения итераций алгоритма.

Расширения $F C M$ - и $G K$-алгоритмов (получаются $E$ - $F C M$ - и $E$ - $G K$-алгоритмы) состоят в следующем [5]. 
В качестве прототипов кластеров используются объемные прототипы(volume prototypes). Объемный прототип - выпуклое и компактное подпространство пространства признаков. В частности, если в алгоритме $E-F C M$ используется евклидова метрика, тогда таким прототипом будет гипершар с центром $v_{i}$ и радиусом $r_{i}$ для $i$-го кластера. Вектор признаков $x_{k}$, для которого расстояние $d_{i k}\left(x_{k}, v_{i}\right) \leq r_{i}$, является элементом объемного прототипа и входит в $i$-й кластер с членством 1.0. В алгоритме $E$ - $G K$ объемный прототип кластера - гиперэллипсоид.

Мера расстояния, используемая в расширенных алгоритмах, определяется на основе исходной меры $d$. Для вектора $x_{k}$ сначала вычисляется значение $d_{i k}\left(x_{k}, v_{i}\right)$, а затем расстояние до объемного прототипа берется равным $\widetilde{d_{l k}}=\max \left(0, d_{i k}-r_{i}\right)$. Таким образом, векторы признаков, лежащие внутри объемного прототипа $i$-го кластера, имеют нулевое значение членства в других кластерах, что уменьшает влияние плотных областей в данных на центры других кластеров. Размеры объемных прототипов определяются на основе объемов кластеров.

Вводится понятие «сходства» (similarity) кластеров. Сходство кластеров определяется на основе нечеткой включающей меры. Для двух нечетких кластеров $u_{i}\left(x_{k}\right)$ и $u_{j}\left(x_{k}\right)$ нечеткая включающая мера определяется как

$$
I_{i j}=\frac{\sum_{k=1}^{N} \min \left(u_{i k}, u_{j k}\right)}{\sum_{k=1}^{N} u_{i k}} .
$$

Здесь $N$ - число векторов признаков в наборе данных; $u_{i k}$ - значение членства $k$-го вектора в $i$-м кластере. Включающая мера не является симметрической, но она используется для построения симметрической меры сходства нечетких кластеров $u_{i}\left(x_{k}\right)$ и $u_{j}\left(x_{k}\right)$ :

$$
S_{i j}=\max \left(I_{i j}, I_{j i}\right) \text {. }
$$

Начиная с заведомо большего числа кластеров, кластеры, степень сходства которых превышает заданный порог, объединяются в итерационном процессе кластеризации для того, чтобы получить подходящее разбиение данных.

Приведенные ниже рисунки демонстрируют процесс построения карты нечеткой кластеризации алгоритмом $E-F C M$. На рис. 1 приведен фрагмент изображения бассейна Обского водохранилища, полученного 19.04.2011 ИС3 Terra (EOS AM-1), сканер Modis. Рис. 2 содержит изображения нечетких кластеров (всего их два), соответствующих состоянию водно-ледовой поверхности бассейна водохранилища.

Заметим, что во входных данных было задано 7 кластеров, в результате работы алгоритма все векторы признаков были разбиты по двум кластерам. В качестве начального разбиения векторов признаков по нечетким кластерам используются выходные данные алгоритма C-средних.

Соответствующая вычислительная процедура реализована в рамках модели параллельного программирования ОреnMP под управлением OC Windows. Включение алгоритмов $E$-FCM и $E-G K$ в состав программного комплекса по обработке спутниковых данных PlanetaMonitoring позволяет расширить возможности системы кластеризации комплекса по построению разбиения, наиболее полно соответствующего внутренней структуре данных.

$$
-725-
$$




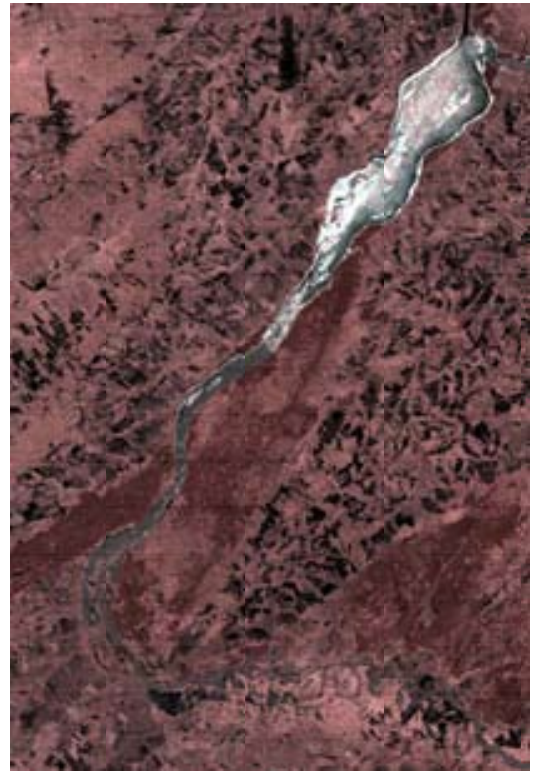

Рис. 1

Fig. 1

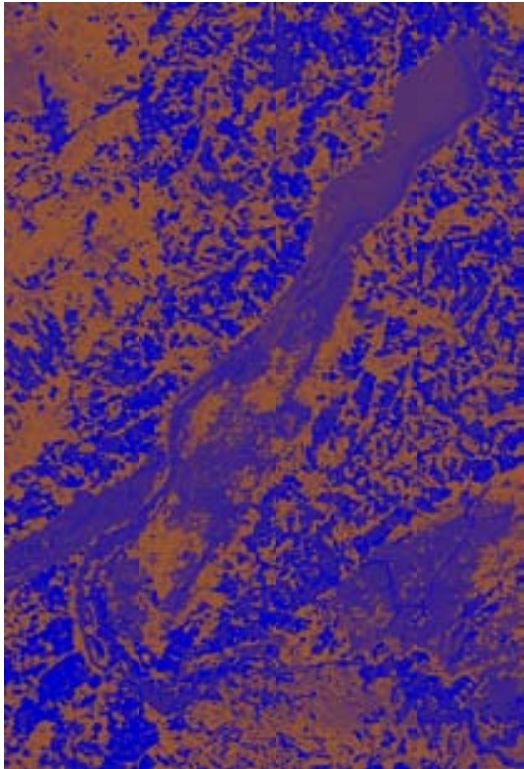

Рис. 2

Fig. 2

Работа выполнена частично при финансовой поддержке Российского фонда фундаментальных исследований (проект № 16-07-00066) и Программы І.33П фундаментальных исследований Президиума РАН (проект № 0315-2015-0012).

\section{Список литературы}

[1] Асмус В.В. Программно-аппаратный комплекс обработки спутниковых данных и его применение для задач гидрометеорологии и мониторинга природной среды. Москва, 2002. 75 с. [Asmus V.V. Software-hardware complex processing of satellite data and its application to problems of hydrometeorology and environmental monitoring, Moskva, 2002. 75 p. (in Russian)]

[2] Шовенгердт Р.А. Дистанционное зондирование. Модели и методы обработки изображений. М.: Техносфера, 2010. 560 c. [Schowengerdt R.A. Remote sensing. Models and methods for image processing. Moskva, Technosfera, 2010, 560 p. (in Russian)]

[3] Bezdek J.C. Pattern recognition with fuzzy objective function algorithms. Plenum Press, New York, 1981.

[4] Асмус В.В., Бучнев А.А., Пяткин В.П. Жесткая и нечеткая кластеризация данных дистанционного зондирования Земли. Журнал СФУ. Сер. Техника и технологии, 2016, 9(7), 972-978. DOI: 10.17516/1999-494X-2016-9-7-972-978. [Asmus V.V., Buchnev A.A., Pyatkin V.P. Hard and fuzzy clustering of the earth remote sensing data. J. Sib. Fed. Univ. Eng. technol., 2016, 9(7), 972-978. DOI: 10.17516/1999-494X-2016-9-7-972-978. (in Russian)]

[5] Uzay Kaimak and Magne Setnes. Extended Fuzzy Clustering Algorithms. ERIM report series ERS-2000-51-LIS. Rotterdam, Netherlands, November 2000, 24. 